\title{
All-Fiber Liquid-Level Sensor Based on In-Line MSM Fiber Structure
}

\author{
Tingting SUN ${ }^{1}$, Zigeng $\mathrm{LIU}^{2}$, Yun LIU², Yang ZHANG ${ }^{2}$, \\ Zhenguo JING ${ }^{2}$, and Wei PENG ${ }^{2 *}$
}

\author{
${ }^{1}$ School of Optoelectronic Engineering and Instrumentation Science, Dalian University of Technology, Dalian 116000, \\ China \\ ${ }^{2}$ School of Physic, Dalian University of Technology, Dalian 116000, China \\ "Corresponding author: Wei PENGＥ-mail: wpeng@dlut.edu.cn
}

\begin{abstract}
We propose and demonstrate an all-fiber liquid-level sensor using an in-line multimode-single-mode-multimode (MSM) fiber structure. A piece of single-mode fiber (SMF) is spliced to two sections of equivalent multimode fiber (MMF) which are used as both mode splitter and mode coupler. The cladding mode will be excited when the light propagates from MMF to SMF, and then it will be combined with fundamental mode to form a Mach-Zehnder interferometer (MZI) when the light propagates from SMF to the other MMF. The liquid level is detected by the selected resonant dips shift of the transmission spectrum. A sensing sensitivity of $264.6 \mathrm{pm} / \mathrm{mm}$ is achieved for the proposed sensor with an SMF length of $26 \mathrm{~mm}$. Due to its compact structure, easy fabrication, and high sensitivity, the proposed liquid-level sensor is attractive for practical applications in a variety of fields, such as marine detection and chemical processing.
\end{abstract}

Keywords: Liquid-level sensor; MSM fiber structure; MZI; optical fiber sensor

Citation: Tingting SUN, Zigeng LIU, Yun LIU, Yang ZHANG, Zhenguo JING, and Wei PENG, "All-Fiber Liquid-Level Sensor Based on In-Line MSM Fiber Structure," Photonic Sensors, 2021, 11(3): 291-297.

\section{Introduction}

In many industrial production processes, it is necessary to measure or monitor the liquid-level information, such as fuel storage system, poisonous liquid collection, and oil transportation. Among many of the liquid-level sensors, electrical methods are wildly investigated due to their high sensitivity and fast response [1, 2]. A grounded capacitive based liquid-level sensor [2] was reported with a high sensitivity of $0.0148 / \mathrm{cm}$ and a fast response time of $20 \mathrm{~ms}$. However, the sensors based on electrical principles are difficult to deal with the liquid-level measurement in severe environments like explosive, conductive, and flammable environments. Another well-known liquid-level measurement method, mechanical method, suffers from its shortcomings of big size, low accuracy, and unsafety [3]. To date, there are many efforts devoted to the development of fiber-optic liquid-level sensors owing to their excellent features, such as high sensitivity, small size, immunity to electromagnetic interference, and resistance to harsh environments [4-6]. Recently, a number of fiber grating sensing structures, including fiber Bragg gratings (FBGs) [7, 8], long period gratings (LPGs) $[9,10]$, and tilted fiber Bragg gratings (TFBGs) [11, 12], have been demonstrated for liquid-level measurement. However, the liquid temperature variation will affect the measurement accuracy. 
Besides, complicated grating fabrication processes limit their applications. Liquid-level sensors based on multimode interference were also proposed [13, 14]. In particular, Li et al. [13] reported a no-core fiber based liquid-level sensor, which held the ability of temperature compensation achieved by an FBG. Likewise, the added FBG increased the fabricated steps of the sensor and then furtherly caused a more complicated sensing structure. Moreover, there are many other liquid-level sensors based on the principle of photonic crystal [15], Fabry-Perot interferometer [16, 17], and peanuts shape Mach-Zehnder interferometer (MZI) [18]. All these sensors are with complex sensor structures and fabrication processes leading to low sensing repeatability, which need further improvement.

In this paper, we propose an in-line multimodesingle-mode-multimode (MSM) fiber structure for the liquid-level measurement. The sensing head is realized by fusion splicing a section of single mode fiber (SMF) between two short multimode fibers (MMFs), of which the fabrication process is quite simple and low-cost. A sensing sensitivity of $264.6 \mathrm{pm} / \mathrm{mm}$ is achieved for the proposed sensor with an SMF length of $26 \mathrm{~mm}$. Besides, the experimental result shows that the proposed sensor has a relatively low temperature sensitivity of $6.63 \mathrm{pm} /{ }^{\circ} \mathrm{C}$ compared with other liquid-level sensors based on the fiber grating. The features of easy fabrication, high sensitivity, and low temperature response make the proposed liquid-level sensor more desirable for real application.

\section{Operation principle}

The structure and the configuration of the proposed sensing head with the MSM fiber structure are shown in Figs. 1(a) and 1(b). The sensing structure is based on an SMF fusion spliced to two pieces of the MMF with the same length. When the sensing light propagates from the lead-in fiber into the MMF, it will excite the high order modes, part of which will be coupled into the cladding of the SMF. Both the cladding modes and the core mode propagating in the SMF will eventually be coupled back to the other MMF and then form an MZI due to the larger core diameter of the MMF corresponding to the SMF. The cladding modes propagating in the SMF is sensitive to the surrounding refractive index (RI). When the SMF section is immersed into the liquid, the surrounding RI will be changed resulting in a shift of spectrum. Therefore, the liquid-level information could be recognized by the resonance peak shift.

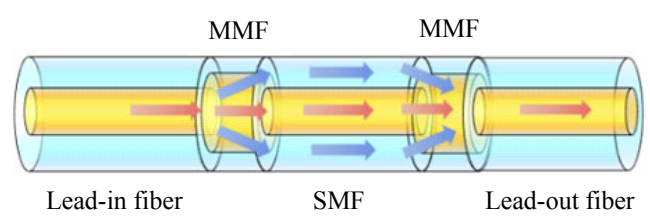

(a)

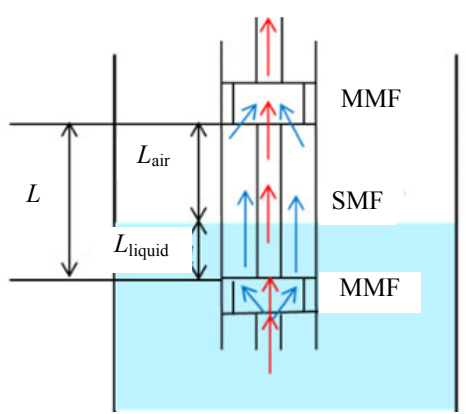

(b)

Fig. 1 Schematic diagrams of (a) the MSM fiber structure and (b) the sensing configuration.

When light transmits from the lead-in SMF to the MMF, high order modes will be excited because of the fiber core mismatch. Assuming the field profile of $m$ th mode within the MMF as $\varphi_{m}$, the field distribution at the MMF can be described as follows [19]

$$
E(r, 0)=\sum_{m=1}^{M} b_{m} \varphi_{m}(r)
$$

where $M$ is the highest order of the MMF modes, $r$ is the path of the light, $E(r, 0)$ is the core mode of the lead-in SMF, and $b_{m}$ is the excitation coefficient of $\varphi_{m}$, which can be expressed as

$$
b_{m}=\frac{\int_{0}^{\infty} E(r, 0) \varphi_{m}(r) r \mathrm{~d} r}{\int_{0}^{\infty} \varphi_{m}(r) \varphi_{m}(r) r \mathrm{~d} r} .
$$

When the light travels in the MMF at a distance of $L_{1}$, the field distribution reads 


$$
E\left(r, L_{1}\right)=\sum_{m=1}^{M} b_{m} \varphi_{m}(r) \exp \left(\mathrm{j} \beta_{m} L_{1}\right)
$$

where $\beta_{m}$ is the propagation constant of the $m$ th mode. As the light transmits into the middle SMF, part of the power is coupled from the core of the MMF to the cladding of the middle SMF forming the forward cladding modes. Assuming the $n$th mode of the middle SMF as $\Phi_{n}(r)(n=1$ for core mode and $n>1$ for cladding modes), then the excitation coefficient of each mode satisfies

$$
C_{n}=\frac{\int_{0}^{\infty} E\left(r, L_{1}\right) \Phi_{n}(r) r \mathrm{~d} r}{\int_{0}^{\infty} \Phi_{n}(r) \Phi_{n}(r) r \mathrm{~d} r} .
$$

After passing through the middle SMF, the amplitude of the $n$th cladding mode can be written as

$$
E_{n}(r)=C_{n} \Phi_{n}(r) \exp \left(\mathrm{j} \beta_{n} L\right)
$$

where $\beta_{n}$ is the propagation constant of the $n$th cladding mode. Therefore, the power of the $n$th mode at the end of the middle SMF can be expressed as follows

$$
I_{n}=\left|E_{n}^{*}(r) \cdot E_{n}(r)\right| .
$$

The cladding mode and the core mode within the middle SMF will interfere in the second MMF, and the interference intensity can be expressed as

$$
\begin{gathered}
I=I_{1}+I_{n}+2 \sqrt{I_{1} I_{n}} \cos \left(\frac{2 \pi \Delta n_{\mathrm{eff}}^{n} L}{\lambda}\right) \\
\Delta n_{\mathrm{eff}}^{n}=n_{\text {core }}-n_{\mathrm{cl}}^{n}
\end{gathered}
$$

where $I_{1}$ and $I_{n}$ are the intensities of the core mode and the cladding mode, respectively; $n_{\text {core }}$ and $n_{\mathrm{cl}}^{n}$ are the effective refractive indices of the core mode and the $n$th cladding mode, respectively. Although many cladding modes are excited in the middle SMF, only one major cladding mode interferes with the core mode, whereas other cladding modes have a minor modulation effect on the main interference [20]. Here, we only consider the main interference mode in (7). The phase difference between the core and the cladding modes can be written as

$$
\frac{2 \pi \Delta n_{\mathrm{eff}}^{n} L}{\lambda_{\mathrm{dip}}}=(2 m+1) \pi \text {. }
$$

Thus, $\lambda_{\text {dip }}$ can be obtained as follows

$$
\lambda_{\text {dip }}=\frac{2 \Delta n_{\mathrm{eff}}^{n} L}{2 m+1} .
$$

Because the cladding modes propagating in the SMF are guided by the cladding boundary, the middle SMF is much sensitive to the surrounding medium [21]. Liquid-level variation will affect the effective refractive index of the cladding mode leading to a drift of the interference fringe. Therefore, the liquid-level information can be monitored by capturing the dip wavelength of the spectrum.

\section{Experiments and results}

The fabrication process of the proposed MSM fiber structure, schematically shown in Fig. 2, consists of 3 steps: (1) two sections of the MMF with a core diameter of $100 \mu \mathrm{m}$ are fusion spliced to both end faces of a piece of the standard SMF with a commercial fusion splicer (Fujikura, FSM-50s); (2) the MMFs are cleaved into $2 \mathrm{~mm}$ length using a fiber cleaver (Furukawa, S325); (3) the fabricated sensing head is spliced to a lead-in fiber and a lead-out fiber for sensing light launching. To reduce the curvature effect of multimode interference induced by the MMFs [22], the length of the MMFs is set as short as possible. In this paper, the length of the MMFs was set to $2 \mathrm{~mm}$. The middle SMF between two short MMFs is the sensing region.

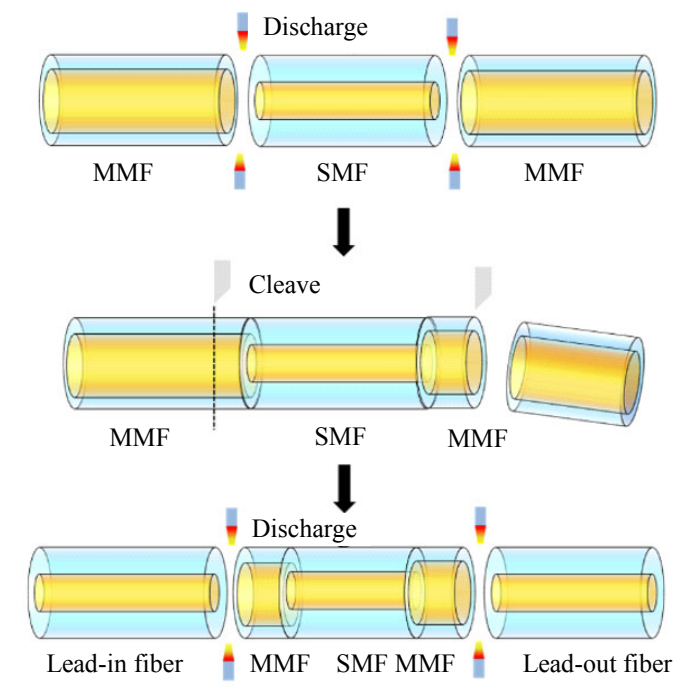

Fig. 2 Outline of the sensor fabrication process. 
The experimental setup for the proposed sensor calibration and testing is illustrated in Fig. 3. The MSM fiber sensor was fastened on a glass rod with two fiber holders in case of undesirable fiber bending. The glass rod was vertically immersed in a water tank, beside which a ruler was fixed for liquid-level calibration. The refractive index of water in this experiment was 1.333. A broadband light source (BBS) provided probing light with an output wavelength range from $1510 \mathrm{~nm}$ to $1590 \mathrm{~nm}$. An optical spectrum analyzer (OSA, Yokogawa, AQ6370) with a wavelength resolution of $0.02 \mathrm{~nm}$ was used to measure the interference spectrum.

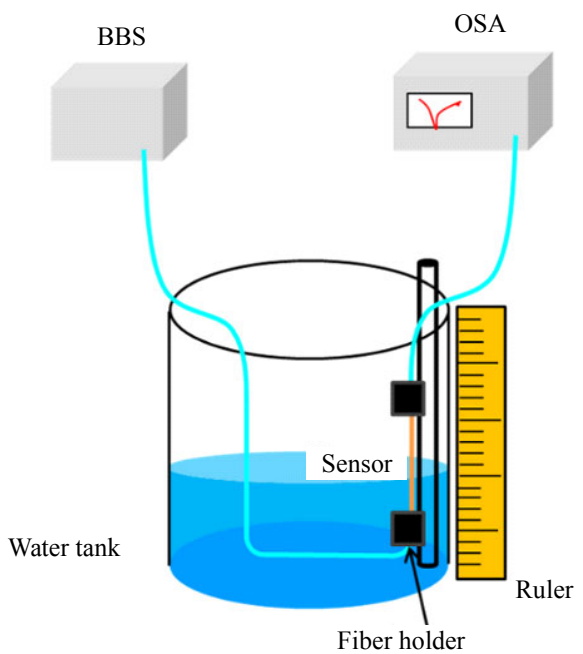

Fig. 3 Schematic of the experimental setup for sensor calibration and testing.

To demonstrate the performance of the proposed sensor, we fabricated three MSM fiber structure sensors with different SMF lengths and measured their responses to the liquid-level. The middle SMF lengths of the three sensors were approximately $23 \mathrm{~mm}, 24 \mathrm{~mm}$, and $26 \mathrm{~mm}$, respectively. A syringe was applied to adjust the liquid-level for the sensor calibration. The liquid-level $L_{\text {liquid }}$ was gradually risen from $0 \mathrm{~mm}$ to $15 \mathrm{~mm}$ at room temperature with a step increment of $3 \mathrm{~mm}$. Figure 4 shows the transmission spectra of three sensors when the sensors were applied with different liquid-levels. The dip wavelengths of the transmission spectra were shifted toward long wavelength. The sensor with the longest middle SMF length $(26 \mathrm{~mm})$ shows the largest wavelength shift $(3.99 \mathrm{~nm})$ with a liquid-level range of $0 \mathrm{~mm}-15 \mathrm{~mm}$.

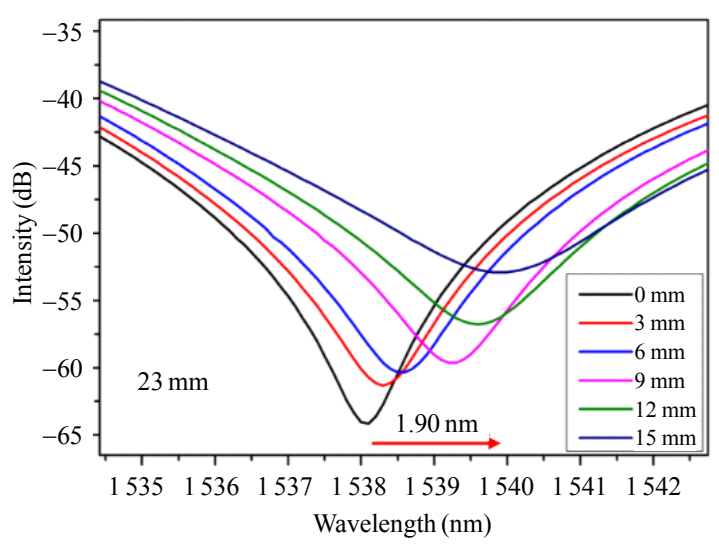

(a)

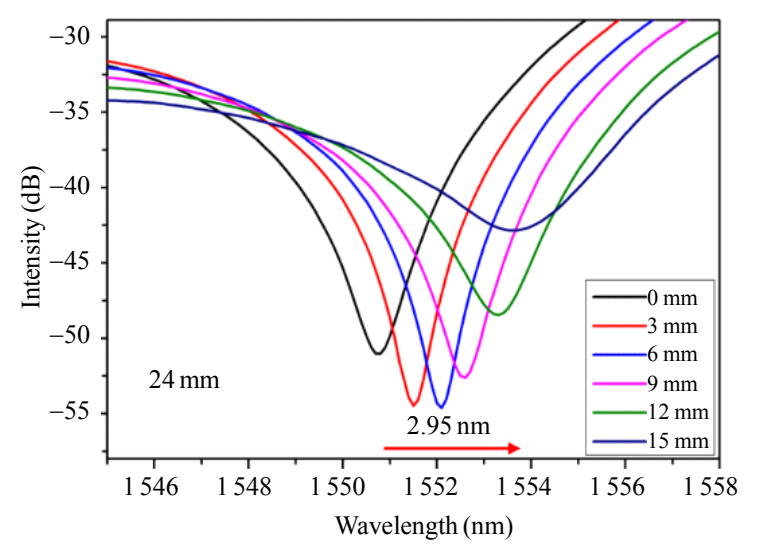

(b)

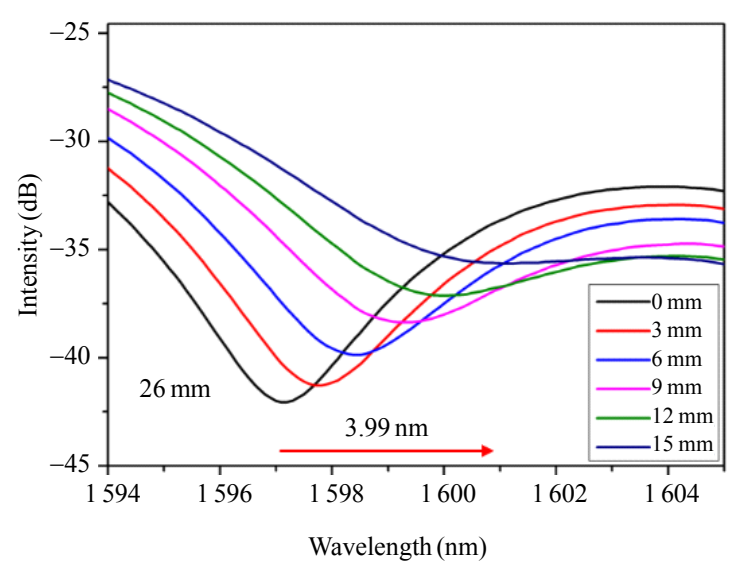

(c)

Fig. 4 Transmission spectra variation with different lengths $L$ of the SMF: (a) $L_{1}=23 \mathrm{~mm}$, (b) $L_{2}=24 \mathrm{~mm}$, and (c) $L_{3}=26 \mathrm{~mm}$.

The wavelength of the interference dip vs. the liquid-level for each sensor is summarized in Fig. 5. 
The dip wavelength as a function of the liquid-level exhibits good linearity, which agrees well with the theoretical prediction [as expressed in (10)]. The sensitivities of $134.4 \mathrm{pm} / \mathrm{mm}, 196.7 \mathrm{pm} / \mathrm{mm}$, and $264.6 \mathrm{pm} / \mathrm{mm}$ were obtained with the sensors of the middle SMF lengths of $23 \mathrm{~mm}, 24 \mathrm{~mm}$, and $26 \mathrm{~mm}$, respectively. The sensitivity calibration results indicate that the sensor with a larger $L$ will perform a higher sensitivity. If the length of the middle SMF is appropriately increased, the sensor sensitivity will be further improved. However, because the cladding mode transmitting in the middle SMF is much sensitive to the surrounding environment such as temperature, refractive index, and strain, overlength SMF will increase the instability of the sensor [21]. Besides, transmission loss of the cladding mode increases with the length of the middle SMF resulting in a fringe visibility reduction of the transmission spectrum. Hence, there is a trade-off between the sensor sensitivity and the sensing performance. Note that the refractive index will decrease the effective refractive index difference between the core and cladding modes when the sensor is immersed in the liquid, which will lead to a slight increase in the sensitivity [18].



Fig. 5 Wavelength shift response to different lengths of the middle SMF.

To characterize the wavelength noise of the proposed liquid-level sensor, the sensor with the $26 \mathrm{~mm}$ length middle SMF was fixed in an environmental chamber with room temperature (around $22^{\circ} \mathrm{C}$ ). The transmission spectra were continuously captured for $4 \mathrm{~s}$ at a frame rate of 300 Hz. The dip wavelength variation of transmission spectra is summarized in Fig. 6. The standard deviation of the wavelength variations of the selected sensing dip was $0.89 \mathrm{pm}$, corresponding to a liquid-level resolution of $3.4 \times 10^{-3} \mathrm{~mm}$ with a liquid-level sensitivity of $264.6 \mathrm{pm} / \mathrm{mm}$. The feature of relatively high sensing resolution guarantees high accuracy of liquid-level measurement, which is highly desirable for real sensing applications.

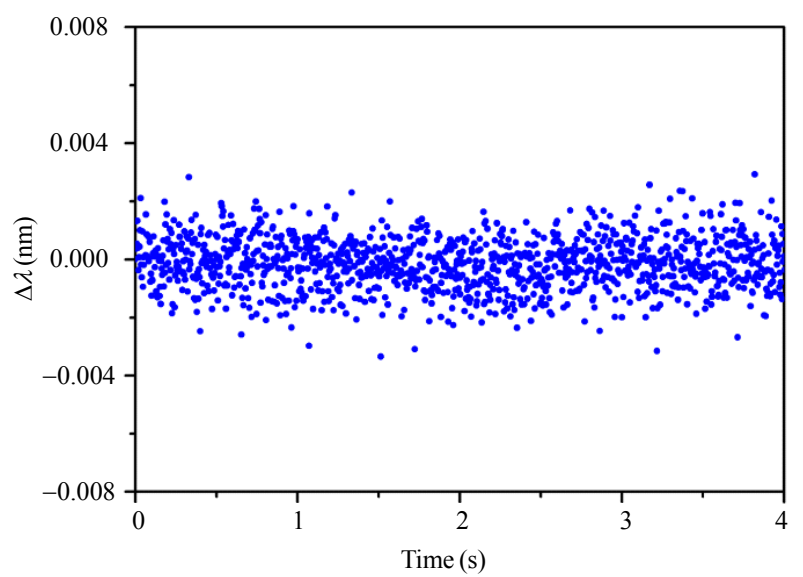

Fig. 6 Dip wavelength variations with time for the sensor placed in the water.

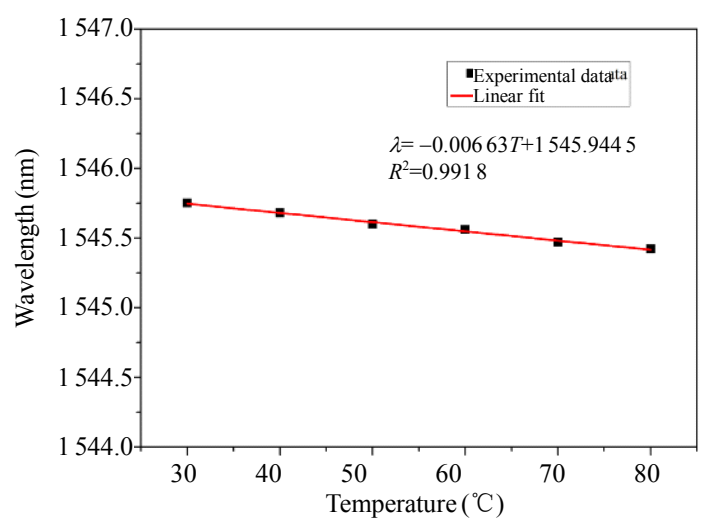

Fig. 7 Sensor response at the wavelength of the transmission dip to different temperatures.

Liquid temperature variation will affect the accuracy of the liquid-level sensors which need to be immersed in the liquid while sensing. Therefore, to investigate the temperature response of the proposed sensor, a sensor with $26 \mathrm{~mm}$ length SMF was tested by fixing it in a water bath with a temperature accuracy of $0.1{ }^{\circ} \mathrm{C}$. The water bath was 
full filled with distilled water which is the same as the one used in the process of liquid-level sensitivity calibration. The liquid temperature in the water bath was adjusted from $30^{\circ} \mathrm{C}$ to $80^{\circ} \mathrm{C}$ with a step size of $10^{\circ} \mathrm{C}$. As shown in Fig. 7, the temperature sensitivity was $6.63 \mathrm{pm} /{ }^{\circ} \mathrm{C}$ for the proposed sensor with $26 \mathrm{~mm}$ length SMF, which could be eliminated in the liquid-level sensing process.

\section{Conclusions}

In summary, an all-fiber liquid-level sensor based on the in-line MSM fiber structure has been proposed and experimentally demonstrated. The sensor was based on the principle of MZI which was originated from the dual MMFs structure to act as both mode coupler and splitter. The cladding mode was excited by the MMF and propagates along with the cladding of the middle SMF, which is sensitive to the surrounding RI. A sensing sensitivity of $264.6 \mathrm{pm} / \mathrm{mm}$ was achieved for the proposed sensor with an SMF length of $26 \mathrm{~mm}$, which exhibited a relatively high resolution of $3.4 \times 10^{-3} \mathrm{~mm}$. Moreover, the temperature sensitivity of the proposed liquid-level sensor was as low as $6.63 \mathrm{pm} /{ }^{\circ} \mathrm{C}$. Compared with conventional liquid-level sensors using electronic sensing elements, the proposed liquid-level sensor possesses many advantages such as small size, immunity to electromagnetic interference, resistance to harsh environments, and remote measurement capability, making it more suitable for many liquid-level sensing applications in harsh conditions.

\section{Acknowledgement}

This study was supported in part by the National Natural Science Foundation of China (NSFC) (Grant Nos. 61727816, 61520106013, and 61705031), in part by China Postdoctoral Science Foundation (Grant Nos. 2017M610175 and 2018T110216), in part by Fundamental Research Funds for Central Universities (Grant No. DUT19LAB32) Sponsor, and financial support acknowledgments are placed here.
Open Access This article is distributed under the terms of the Creative Commons Attribution 4.0 International License (http://creativecommons.org/licenses/by/4.0/), which permits unrestricted use, distribution, and reproduction in any medium, provided you give appropriate credit to the original author(s) and the source, provide a link to the Creative Commons license, and indicate if changes were made.

\section{References}

[1] K. Shannon, X. Li, Z. Wang, and J. D. N. Cheeke, "Mode conversion and the path of acoustic energy in a partially water-filled aluminum tube," Ultrasonics, 1999, 37(4): 303-307.

[2] F. Reverter, X. J. Li, and G. C. M. Meijer, "Liquid-level measurement system based on a remote grounded capacitive sensor," Sensors and Actuators A: Physical, 2007, 138(1): 1-8.

[3] B. W. Northway, N. H. Hancock, and T. Tran-cong, "Liquid level sensors using thin walled cylinders vibrating in circumferential modes," Measurement Science \& Technology, 1995, 6(1): 85

[4] Y. S. Zhang, W. G. Zhang, L. Chen, Y. X. Zhang, S. Wang, and T. Y. Yan, "High sensitivity optical fiber liquid level sensor based on compact MMF-HCF-FBG structure," Measurement Science and Technology, 2018, 29(5): 055104.

[5] N. S. Fabián, A. B. Socorro-Leránoz, I. D. Villar, S. Díaz, and I. R. Matías, "Multimodecoreless-multimode fiber-based sensors: theoretical and experimental study," Journal of Lightwave Technology, 2019, 37(15): 3844-3850.

[6] K. Iwamoto and I. Kamata, "Liquid-level sensor with optical fibers," Applied Optics, 1992, 31(1): 51-54.

[7] T. Guo, Q. D. Zhao, Q. Y. Dou, H. Zhang, L. F. Xue, G. L. Ling, et al., "Temperature-insensitive fiber Bragg grating liquid-level sensor based on bending cantilever beam," IEEE Photonics Technology Letters, 2005, 17(11): 2400-2402.

[8] K. R. Sohn and J. H. Shim, "Liquid-level monitoring sensor systems using fiber Bragg grating embedded in cantilever," Sensors and Actuators A: Physical, 2009, 152(2): 248-251.

[9] H. Y. Fu, X. W. Shu, A. P. Zhang, W. S. Liu, L. Zhang, S. L. He, et al., "Implementation and characterization of liquid-level sensor based on a long-period fiber grating Mach-Zehnder interferometer," IEEE Sensors Journal, 2011, 11(11): 2878-2882.

[10] Y. Huang, B. K. Chen, G. D. Chen, H. Xiao, and S. U. Khan, "Simultaneous detection of liquid level and refractive index with a long-period fiber grating based sensor device," Measurement Science and 
Technology, 2013, 24(9): 095303.

[11] B. B. Gu, W. L. Qi, Y. Y. Zhou, Z. F. Wu, P. P. Shum, and F. Luan, "Reflective liquid level sensor based on modes conversion in thin-core fiber incorporating tilted fiber Bragg grating," Optics Express, 2014, 22(10): 11834-11839.

[12] T. Osuch, T. Jurek, K. Markowski, and K. Jedrzejewski, "Simultaneous measurement of liquid level and temperature using tilted fiber Bragg grating," IEEE Sensors Journal, 2015, 16(5): 1205-1209.

[13] C. Li, T. G. Ning, C. Zhang, J. Li, X. D. Wen, L. Pei, et al., "Liquid level measurement based on a no-core fiber with temperature compensation using a fiber Bragg grating," Sensors and Actuators A: Physical, 2016, 245: 49-53.

[14] Y. Q. Wen, L. Xia, Y. L. Ran, J. Rohollahnejad, and D. M. Liu, "All-fiber in-line SNS structure liquid level sensor," Asia Communications and Photonics Conference 2015, Hong Kong, Nov. 19-23, 2015.

[15] H. Y. Choi, M. J. Kim, and B. H. Lee, "All-fiber Mach-Zehnder type interferometers formed in photonic crystal fiber," Optics Express, 2007, 15(9): $5711-5720$.

[16] T. Lü and S. Yang, "Extrinsic Fabry-Perot cavity optical fiber liquid-level sensor," Applied Optics, 2007, 46(18): 3682-3687.
[17] C. W. Lai, Y. L. Lo, J. P. Yur, W. F. Liu, and C. H. Chuang, "Application of Fabry-Perot and fiber Bragg grating pressure sensors to simultaneous measurement of liquid level and specific gravity," Measurement, 2012, 45(3): 469-473.

[18] M. M. Sun, Y. X. Jin, and X. Y. Dong, “All-fiber Mach-Zehnder interferometer for liquid level measurement," IEEE Sensors Journal, 2015, 15(7): 3984-3988.

[19] Q. Wu, Y. Semenova, B. B. Yan, Y. Q. Ma, P. F. Wang, C. X. Yu, et al., "Fiber refractometer based on a fiber Bragg grating and single-modemultimode-single-mode fiber structure," Optics Letters, 2011, 36(12): 2197-2199.

[20] Z. L. Xu, Q. Z. Sun, J. H. Wo, Y. Dai, X. L. Li, and D. M. Liu, "Volume strain sensor based on spectra analysis of in-fiber modal interferometer," IEEE Sensors Journal, 2013, 13(6): 2139-2145.

[21] J. H. Wo, Q. Z. Sun, H. Liu, X. L. Li, J. J. Zhang, D. M. Liu, et al., "Sensitivity-enhanced fiber optic temperature sensor with strain response suppression," Optical Fiber Technology, 2013, 19(4): 289-292.

[22] Y. Gong, T. Zhao, Y. J. Rao, and Y. Wu, "All-fiber curvature sensor based on multimode interference," IEEE Photonics Technology Letters, 2011, 23(11): 679-681. 